Eléments et éphéméride de la comète 1906 a.

J'ai l'honneur de communiquer les éléments et l'éphéméride de la nouvelle comète 1906 a calculés à l'aide des observations suivantes:

\begin{tabular}{|c|c|c|c|c|}
\hline 1906 & t. m. local & $\alpha$ app. & $\delta$ app. & lieu \\
\hline nv. 29 & $6^{\mathrm{h}} 48^{\mathrm{m}} \cdot 5$ & $244^{\circ} 24^{\prime} 57^{\prime \prime}$ & $+51^{\circ} \quad 7^{\prime} 40^{\prime \prime}$ & Strasbourg \\
\hline 30 & 9.6 & $244 \quad 4 \quad 55$ & +531927 & Utrecht \\
\hline $3 \mathrm{I}$ & 1659.5 & $243 \quad 42 \quad 28$ & +552441 & Lyon \\
\hline
\end{tabular}

$$
\left.\begin{array}{rl}
T & =\text { r } 906 \text { Janv. } 0.5686 \text { t. m. Paris } \\
\infty & =\text { I00 } 57^{\prime} 37^{\prime \prime} \\
\delta & =2893^{6} 3^{8} \\
i & =\text { r24 } 593^{\mathrm{I}}
\end{array}\right\} \text { I906.0 }
$$

Représentation du lieu moyen $(\mathrm{O}-\mathrm{C})$ :

\begin{tabular}{|c|c|c|c|c|c|}
\hline 1906 & $\alpha$ app. & $\delta$ app. & $\log r$ & $\log A$ & Eclat \\
\hline Évr. Io & $15^{\mathrm{h}} 30^{\mathrm{m}} 8^{\mathrm{s}}$ & $+75^{\circ} 31: 3$ & 0.1649 & 9.9359 & I. 2 \\
\hline I 2 & $1454 \quad 44$ & +7943.3 & 0.1686 & $9.934^{6}$ & $\mathrm{I} .2$ \\
\hline 14 & 133512 & $+8321 \cdot 5$ & 0.1723 & $9.936 \mathrm{r}$ & I. I \\
\hline I 6 & 104124 & $+84 \cdot 55.8$ & 0.1761 & $9.94 \circ 3$ & I.I \\
\hline
\end{tabular}
$\cos \beta \mathrm{d} \lambda=-5^{\prime \prime} \mathrm{d} \beta=+\mathrm{I}^{\prime \prime}$

Ephéméride pour I $2^{h}$ t. m. Paris.

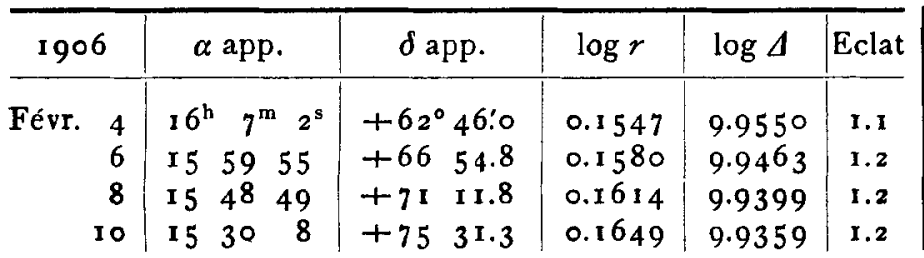

L'éclat du 29 janvier a été pris comme unité.

Observatoire de Paris, le 5 février 1906.

\begin{tabular}{|c|c|c|c|c|c|c|c|c|c|c|c|}
\hline \multirow{2}{*}{$\frac{1906}{\text { Febr, } 20}$} & $\alpha$ vera & $\delta$ vera & \multirow{2}{*}{$\frac{\log r}{0.2001}$} & \multirow{2}{*}{$\frac{\log \Delta}{9.99 \mathrm{r} 2}$} & \multirow{2}{*}{$\frac{\mathrm{H}}{0.88}$} & 1906 & $\alpha$ vera & $\delta$ vera & \multirow{2}{*}{$\frac{\log r}{0.2282}$} & \multirow{2}{*}{$\frac{\log \Delta}{0.0748}$} & \multirow{2}{*}{$\begin{array}{c}\mathrm{H} \\
0.53\end{array}$} \\
\hline & $7^{\mathrm{h}} 32^{\mathrm{m}} 10^{\mathrm{s}}$ & $+8 I^{\circ} 42: 8$ & & & & März 4 & $5^{\mathrm{h}} 48^{\mathrm{m}} 5^{0^{\mathrm{s}}}$ & $+63^{\circ}$ 1 $8: 8$ & & & \\
\hline 2 I & 7443 & $\begin{array}{ll}80 & 8.5\end{array}$ & & & & 5 & 4720 & $62 \quad 1.6$ & & & \\
\hline 22 & $\begin{array}{lll}6 & 45 & 35\end{array}$ & $78 \quad 30.2$ & 0.2047 & 0.0024 & 0.82 & 6 & $46 \quad 7$ & 6047.1 & 0.2329 & 0.0909 & $0.4^{8}$ \\
\hline 23 & 3147 & $765 \mathrm{I.t}$ & & & & 7 & 45 & $5935 \cdot 3$ & & & \\
\hline 24 & 2129 & 7512.0 & 0.2 & 0.0 & 0.76 & 8 & $44 \quad 18$ & 5826.1 & 0.2376 & 0.1072 & 0.43 \\
\hline 25 & 1338 & 7334.6 & & & & 9 & $433^{8}$ & $57 \quad 19.3$ & & & \\
\hline 26 & 728 & 7158.9 & 0.2140 & 0.0288 & 0.69 & 10 & 436 & $56 \times 5.0$ & 0.2423 & 0.1236 & 0.39 \\
\hline 27 & $\begin{array}{lll}6 & 2 & 34\end{array}$ & $70 \quad 25.6$ & & & & I I & $424 I$ & 55 I 3. I & & & \\
\hline 28 & $\begin{array}{lll}5 & 5^{8} & 3^{8}\end{array}$ & $\begin{array}{ll}68 & 54.8\end{array}$ & 0.2187 & 0.0435 & 0.64 & 12 & 4222 & 54 I 3.4 & 0.2470 & 0.1398 & $0.3^{6}$ \\
\hline Iärz I & $55 \quad 24$ & 6726.7 & & & & I 3 & $4^{2} 10$ & $53 \times 5.8$ & & & \\
\hline 2 & $5^{2} 47$ & $\begin{array}{ll}66 & 1.3\end{array}$ & 0.2234 & 0.0589 & $0.5^{8}$ & I 4 & $42 \quad 2$ & $5^{2} \quad 20.2$ & 0.25 I 7 & 0.1559 & 0.33 \\
\hline 3 & 5037 & 6438.7 & & & & $\mathbf{I}$ & 4 I $5^{8}$ & $\begin{array}{ll}51 & 26.8\end{array}$ & & & \\
\hline 4 & $\begin{array}{lll}5 & 48 & 50\end{array}$ & $+63 \times 8.8$ & 0.2282 & $0.074^{8}$ & 0.53 & 16 & $\begin{array}{lll}54158\end{array}$ & +5035.3 & 0.2564 & 0.1718 & 0.30 \\
\hline
\end{tabular}

E. Maubant.

Ephemeride des Kometen 1906 a.

Fortsetzung zu Nr. 4073. I $2^{\text {h }}$ M. Z. Berlin.

Korrektion der Ephemeride: Febr. I Rom $+3^{\mathrm{s}}$ o:o, Febr. 2 Rom $+5^{\mathrm{s}}+0^{\prime} 7$.

Kiel, Bureau der Astr. Nachr., I 906 Febr. 12.

\title{
Amerikanische Beobachtungen des Kometen 1906 a
} mitgeteilt von Prof. E. C. Pickering.
Jan. $27.8920 \mathrm{Gr} . \quad \alpha$ app. $=16^{\mathrm{h}} 18^{\mathrm{m}} 49^{\mathrm{s}} .7 \quad \delta$ app. $=+48^{\circ} 5^{\mathrm{I}^{\prime}} \mathrm{1} 5^{\prime \prime} \quad$ Maddrill, Lick Obs. $27.963^{2} \nabla *=161846.4 \%=+4858$ 20 Barnard, Yerkes Obs.

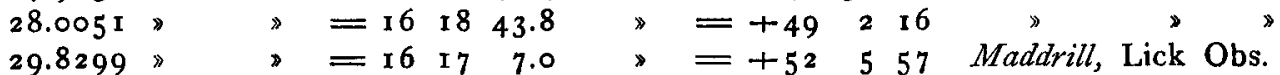 28.93679 Gr. $\Delta \alpha=-2^{\mathrm{m}_{3} 3^{5} 6} \quad \Delta \delta=+\mathrm{x}^{\prime} 1^{\prime \prime} \quad *=\mathrm{BD}$. $+50^{\circ 2282}$ Morgan, Glasgow. $29.00272 \% 》=-014.27 \%=+221=$ BD. +50.2278 Duncan, Flagstaff.

Inhalt zu Nr. 4075. E. E. Barnard. Periodical changes in the size of the glow surrounding the lunar crater Linne. 293. - K. Sotome. Eine Bemerkung über die Bestimmung der Kreisbahn eines kleinen Planeten. 295. - Notiz betr. den Stern BD. + 19.2856. 297. - A. Bemporad. Notizia riguardante la stella AG. Harvard 1461. 299. - A. Antoniazzi. Osservazioni di pianeti e della cometa I905 III fatte a Padova. 299. - L. Gabba. Osservazioni di piccoli pianeti. 303. - Beobachtungen des Kometen I 906 a. 303. - V. Fontana. Elementi ed effemeride del pianeta (5 I6) Amherstia. 305. - E. Maubant: Eléments et éphéméride de la comète I906 a. 307. - M. Ebell. Ephemeride des Kometen 1906 a. 307. - E. C. Pickering. Amerikanische Beobachtungen des Kometen 1906 a. 307 . 